\title{
Psychometric Properties of a Short Korean Version of the Revised Obsessive Intrusion Inventory
}

\author{
Jang-Won Seo ${ }^{1}$, Min-Jung Baek², Mi-So Lee ${ }^{3}$, Ju-Ri Jeon ${ }^{4}$, and Seok-Man Kwon ${ }^{1}$ \\ ${ }^{1}$ Department of Psychology, Seoul National University, Seoul, Republic of Korea \\ ${ }^{2}$ Department of Psychiatry, Yeonsei Feel Hospital, Seoul, Republic of Korea \\ ${ }^{3}$ Department of Psychiatry, Seoul National University Hospital, Seoul, Republic of Korea \\ ${ }^{4}$ Department of Psychiatry, Samsung Medical Center, Seoul, Republic of Korea
}

Objective The Revised Obsessive Intrusion Inventory (ROII) is a 52-item scale that evaluates obsessional intrusive thoughts. The aim of the present study was to validate a short, 20-item Korean version of the ROII (ROII-20).

Methods Of the 1125 participants who completed the ROII-20, 895 participants completed the scale to examine the factor structure of the scale. A subgroup of these participants $(n=53)$ completed the scale twice to determine test-retest reliability. To establish external validity, 230 participants completed the scale and other questionnaires.

Results Exploratory factor analyses suggested a hierarchical model comprising two higher order factors of autogenous obsessions (resulting from aggressive thoughts and sexual thoughts) and reactive obsessions (resulting from thoughts about contamination, thoughts about accidents, and thoughts about dirt). Confirmatory factor analyses supported this model. The results indicated good internal consistency and test-retest reliability. External validity was supported by relationships with obsessive-compulsive symptoms and general distress.

Conclusion The ROII-20 presents good psychometric properties and may be considered as a promising instrument for measuring obsessional intrusions.

Psychiatry Investig 2015;12(3):288-294

Key Words Mental intrusions, Mental intrusions questionnaire, Autogenous obsessions, Reactive obsessions, Reliability, Validity.

\section{INTRODUCTION}

Obsessions are recurrent and persistent thoughts, impulses, or images that are experienced as intrusive and inappropriate, causing marked anxiety or distress. ${ }^{1}$ Obsessions are one of the major symptoms of obsessive-compulsive disorder (OCD), and it has been suggested that obsession-like thoughts can be observed in nonclinical populations as well. ${ }^{2}$ Mental intrusions or intrusive thoughts are the terms that have been used for nonclinical obsession-like thoughts. These thoughts are similar in form and content to clinical obsessions, but the latter are more frequent, anxiety-provoking, and uncontrollable

Received: June 15, 2014 Revised: September 17, 2014

Accepted: September 23, 2014 Available online: July 6, 2015

$\triangle$ Correspondence: Jang-Won Seo, MA

Department of Psychology, Seoul National University, 1 Gwanak-ro, Gwanakgu, Seoul 151-742, Republic of Korea

Tel: +82-2-880-6431, Fax: +82-2-880-6085, E-mail: jangw.seo@gmail.com

(a) This is an Open Access article distributed under the terms of the Creative Commons Attribution Non-Commercial License (http://creativecommons.org/licenses/bync/3.0) which permits unrestricted non-commercial use, distribution, and reproduction in any medium, provided the original work is properly cited. than mental intrusions of nonclinical individuals. ${ }^{3}$ It has been suggested that a nonclinical mental intrusion escalates to a clinical obsession when individuals appraise the mental intrusion as having important meaning to them (e.g., potential threats) and try to reduce the anxiety induced by the negative appraisals with specific neutralizing responses. ${ }^{4}$ The more individuals appraise certain intrusive thoughts as meaningful to themselves and try to control those thoughts, the more they experience the mental intrusions. ${ }^{5}$ The frequency of mental intrusions is an important index of the possibility that a nonclinical mental intrusion escalates to a clinical obsession, ${ }^{5}$ and therefore, properly measuring the frequency of mental intrusions in nonclinical individuals is crucial for preventing manifestations of OCD.

Another important factor that should be considered is the content of mental intrusions. Mental intrusions have various contents (e.g., aggressive thoughts, sexual thoughts, contamination related thoughts, and accident-related thoughts), which are related to cognitive appraisals and neutralization strategies. ${ }^{6}$ However, there are few models that have consid- 
ered the contents of mental intrusions. A taxonomic model proposed by Lee and Kwon ${ }^{6}$ is unique in that it classifies mental intrusions/obsessions based on the differences in their contents, cognitive appraisals, neutralization strategies, trigger stimuli, and the perceived rationality of the thought contents. The model classifies mental intrusions into two subtypes: autogenous obsessions (AOs) and reactive obsessions (ROs). AOs are highly aversive and unrealistic mental intrusions that usually take the form of thoughts, images, or impulses. The themes of AOs are centered primarily on unacceptable aggression, sexual behavior, and blasphemy. Further, AOs tend to occur without clearly perceived antecedents. ROs, in contrast, are relatively realistic mental intrusions that usually take the form of thoughts, doubts, or concerns about contamination, mistakes, accidents, asymmetry, or disarray. Relative to AOs, ROs are more prone to be triggered by external cues, which correspond to specific core threats. Since the AO-RO model was proposed, research has demonstrated meaningful differences between the two subtypes of mental intrusions in several important domains relevant to OCD: 1) subsequent cognitive appraisals, inferences, and neutralization strategies; $^{6,7}$ 2) associated personality features; ${ }^{8,9}$ 3) responsiveness to cognitive therapy; ${ }^{10} 4$ ) distinct neural abnormality. ${ }^{11}$

This evidence suggests that contents of mental intrusions, or at least the subtype of mental intrusions (i.e., AOs vs. ROs), should be considered when measuring mental intrusions. The Revised Obsessive Intrusion Inventory (ROII) ${ }^{2}$ is a well-developed measure that assesses an individual's mental intrusions in terms of content and frequency and has been used in many studies related to mental intrusions and obsessions. The ROII consists of two sections. ${ }^{2}$ In section I, respondents rate how frequently they experience each of 52 intrusive thoughts on a seven-point Likert scale. 52 items of the ROII assess mental intrusions with various contents (e.g., aggressive thoughts/ images/impulses, sexual thoughts, contamination related thoughts, thoughts about accidents and mistakes, thoughts about dirt and cleaning). Section II consists of several items inquiring about mental-intrusion-related cognitive appraisals, emotions, and neutralization strategies. Lee and Kwon ${ }^{6}$ conducted factor analysis on a Korean version of the ROII section I and found that the two-factor structure (AOs and ROs) was appropriate for the scale. This two-factor structure was supported in another study as well. ${ }^{12}$

Although the ROII is a reliable and valid measure for assessing mental intrusions in terms of content and frequency, it still has several limitations. First, the ROII is composed of 52 items, which is relatively time-consuming to complete and could hinder its use both in research and clinical contexts. Second, it addresses various thought contents but certain contents are assessed in much greater proportion than others: 41 items assess AOs and 11 items assess ROs. ${ }^{2}$ Even among items tapping AOs, there is an imbalance of thought contents (i.e., only 10 items for sexual thoughts and 31 items for aggressive thoughts). ${ }^{2}$

Considering these limitations of the ROII, the aim of the current study was to develop a short version of the ROII assessing mental intrusions with a balanced proportion of thought contents. To this end, we examined the psychometric properties (factor structure, internal consistency, test-retest reliability, and external validity) of the short, 20-item Korean version of the ROII (ROII-20).

\section{METHODS}

\section{Participants}

The sample comprised 1125 undergraduate students (50.1\% female) from two universities in Seoul. The students participated in this study in partial fulfillment of their research participation credit. Only native Korean speakers were retained for the study. The age of the participants ranged from 17 to 35 years $($ mean $=21.86$ years, $\mathrm{SD}=2.47)$.

\section{Procedure}

Groups of 50 to 100 individuals completed the ROII-20 and other measures in their university classes. The questionnaires were completed anonymously, and all participants provided informed consent. One subgroup of participants ( $n=$ 431) completed the ROII-20 for Exploratory Factor Analyses (EFA). Some participants in this subgroup $(n=52)$ completed the ROII-20 twice (with an interval of four weeks) to establish test-retest reliability. A personal code instead of an identity was used to monitor these participants across sessions. A second subgroup of participants $(n=464)$ completed the scale for Confirmatory Factor Analyses (CFA). A third subgroup of participants $(n=230)$ completed the ROII-20 and other self-report measures to determine external validity of the ROII-20.

\section{Instruments}

\section{The Revised Obsessive Intrusion Inventory-20}

The ROII-20 is a 20 -item scale that evaluates two different subtypes of mental intrusions labeled as AOs and ROs. All items are scored on a Likert scale from 0 ("never") to 6 ("frequently during the day"). The items of the ROII-20 were taken from the 52-item Korean version of the ROII. ${ }^{6}$ Lee and Kwon $^{6}$ translated the original version of the ROII into Korean and conducted factor analysis on the Korean version of the ROII. To create the ROII-20, we considered two aspects of the Korean version of the ROII: the factor loading and the content. First, we adapted all items with contents related to mis- 
takes and accidents of the scale (items 22, 23, and 24). Second, we adapted all items with contents related to dirt, ordering, and cleaning of the scale (items 50, 51, and 52). Third, we selected four items with contents related to fatal contamination of the scale (items 46, 47, 48, and 49). An item with this content category (item 45 'catching sexual transmitted disease') was removed from further analysis because it had an item-redundancy problem with item 48 ('fatal disease, stranger') and had the lowest factor loading (0.51). Fourth, we selected five items with aggressive contents of the scale that loaded most strongly on the AOs factor (items 12, 16, 21, 27, and 31). We expected that five items would constitute a minimum scale length that would still exhibit acceptable levels of internal consistency ${ }^{13}$ Fifth, we also selected five items with sexual contents of the scale that loaded most strongly on the AOs factor (items 35, 36, 41, 42, and 43).

\section{The Obsessive-Compulsive Inventory-Revised}

The Obsessive-Compulsive Inventory-Revised (OCI-R) is a well-established 18-item questionnaire assessing the severity of OCD symptoms with good psychometric properties: internal consistency, test-retest reliability, and convergent validity. ${ }^{14}$ A Korean version of the OCI-R was administered, which has demonstrated good psychometric properties. ${ }^{15}$

\section{The Padua Inventory-Washington State University Revision}

The Padua Inventory-Washington State University Revision (PIWSUR) is a 39-item questionnaire that assesses the severity of OCD symptoms with good reliability and validity. ${ }^{16}$ We used the obsessional impulses subscale from a Korean version of the PI-WSUR ${ }^{17}$ in this study.

\section{The Centers for Epidemiologic Studies Depression Scale}

The Centers for Epidemiologic Studies Depression Scale (CES-D) is a 20-item questionnaire assessing depressive symptoms with good psychometric properties. ${ }^{18}$ A Korean version of the CES-D was administered, which has demonstrated good reliability and validity. ${ }^{19}$

\section{The Beck Anxiety Inventory}

The Beck Anxiety Inventory (BAI) is a widely used 21-item questionnaire assessing anxiety symptoms. ${ }^{20} \mathrm{~A}$ Korean version of the BAI was administered, which has demonstrated good psychometric properties. ${ }^{21}$

\section{Statistical analyses}

To examine the factor structure of the ROII-20, we conducted EFA with maximum likelihood extraction and direct quartimin rotation. We also performed CFA with $\mathrm{R}$ (version
3.0.1, lavaan package $)^{22}$ to cross-validate the results obtained from EFA. Goodness of fit was tested with $\chi^{2}$, and two other indices were computed: the root mean square error of approximation (RMSEA) and the standardized root mean square residual (SRMR). ${ }^{23}$ An RMSEA of between 0 and 0.05 indicates a good fit, and a value between 0.05 and 0.08 indicates an acceptable fit. An SRMR of between 0 and 0.05 indicates a good fit, and a value between 0.05 and 0.10 indicates an acceptable fit. $^{24}$ We also reported the comparative fit index (CFI). ${ }^{25} \mathrm{~A}$ CFI greater than 0.90 indicates an acceptable fit. The internal reliability of the ROII-20 was examined with the Cronbach a coefficient. Two-tailed Pearson correlations were used to examine relations between the ROII-20 and the other self-report measures included in this study. Pearson correlations were also used to examine the test-retest reliability of the ROII-20. Pairwise treatment of missing data was used.

\section{RESULTS}

\section{Factor structure and reliability of the ROII-20}

Of the 1125 participants, 51 had one item or more missing after completion of the questionnaires and were removed from the analyses. EFA was conducted with 410 participants. These participants were divided into two subgroups. The lower-order EFA was conducted with a subgroup $(n=305)$ to examine the lower-order factor structure of the ROII-20. Eigenvalues of the correlation matrix suggested a five-factor structure (five eigenvalues $>1.0$ ). The five-factor model showed an acceptable fit (RMSEA=0.07) and explained $71.5 \%$ of the total variance. The detailed factor loadings are reported in Table 1. The five factors were consistent with the major thought contents that we aimed to assess using the scale: aggressive thoughts, sexual thoughts, thoughts about contamination, thoughts about mistakes/accidents, and thoughts about dirt/ordering/cleaning. We then conducted the higher-order EFA with another subgroup $(n=105)$. In this step of analysis, we used factor scores of the five-factor model from the lower-order EFA to extract higher-order latent variables. Eigenvalues of the correlation matrix suggested a two-factor structure ( 2 eigenvalues $>1.0$ ). The two-factor model showed a good fit (RMSEA <0.01) and explained $69.4 \%$ of the total variance. Detailed factor loadings are reported in Table 2. The two factors were consistent with the two major subtypes of mental intrusions: AOs and ROs.

We conducted CFA with 434 participants to confirm the factor structure of the ROII-20. The hierarchical model that we obtained from the EFA was tested by maximum likelihood estimation. The results showed that the hierarchical model had an acceptable fit $\left[\chi^{2}(164)=488.43, \mathrm{p}<0.001 ; \mathrm{RMSEA}=0.07\right.$; $\mathrm{SRMR}=0.05 ; \mathrm{CFI}=0.93$ ]. This model is depicted in Figure 1.

Mean, SDs, internal consistency coefficient (Cronbach $\alpha$ ), 
Table 1. Results of the lower order Exploratory Factor Analysis on the ROII-20

\begin{tabular}{|c|c|c|c|c|c|}
\hline Items & Factor 1 & Factor 2 & Factor 3 & Factor 4 & Factor 5 \\
\hline 12. Pushing strangers-train, car & 0.03 & -0.01 & 0.08 & 0.65 & -0.02 \\
\hline 16. Bumping into people & -0.07 & 0.10 & 0.01 & 0.82 & -0.13 \\
\hline 21. Stabbing family members & 0.04 & -0.11 & -0.04 & 0.61 & 0.10 \\
\hline 22. Heat/stove on, accident & -0.01 & 0.04 & 0.81 & -0.01 & -0.05 \\
\hline 23. Home unlocked, intruder & 0.11 & 0.01 & 0.77 & -0.05 & 0.02 \\
\hline 24. Taps left on, flood & -0.06 & 0.00 & 0.50 & 0.16 & 0.13 \\
\hline 27. Throwing something & 0.16 & 0.03 & 0.02 & 0.53 & 0.07 \\
\hline 31. Wrecking something & 0.13 & 0.10 & 0.12 & 0.55 & 0.04 \\
\hline 35. Sex with unacquainted person & 0.78 & 0.08 & 0.11 & -0.08 & -0.08 \\
\hline 36. Sex with authority figure & 0.76 & -0.06 & 0.05 & -0.02 & 0.02 \\
\hline 41. Authority figure naked & 0.72 & -0.04 & -0.01 & -0.02 & 0.12 \\
\hline 42. Stragers naked & 0.70 & 0.03 & -0.06 & 0.26 & -0.03 \\
\hline 43. Sex in public & 0.64 & 0.08 & -0.09 & 0.26 & -0.03 \\
\hline 46. Contamination, door-knobs & 0.00 & -0.01 & 0.02 & -0.05 & 0.83 \\
\hline 47. Contamination, phones & 0.00 & 0.05 & -0.03 & 0.01 & 0.85 \\
\hline 48. Fatal disease, stranger & -0.03 & 0.03 & 0.04 & -0.02 & 0.81 \\
\hline 49. Transmitting fatal disease & 0.05 & 0.12 & 0.05 & 0.08 & 0.68 \\
\hline 50. Everything away & 0.00 & 0.87 & -0.03 & -0.01 & 0.07 \\
\hline 51. All dust off floor & 0.01 & 0.95 & 0.00 & -0.02 & 0.04 \\
\hline 52. Dirt in unseen places & -0.02 & 0.85 & 0.06 & 0.02 & 0.02 \\
\hline
\end{tabular}

ROII-20: Revised Obsessive Intrusion Inventory-20

Table 2. Results of the higher order Exploratory Factor Analysis on the ROII-20

\begin{tabular}{lcc}
\hline \multicolumn{1}{c}{ Lower order factors } & Factor 1 & Factor 2 \\
\hline Sexual thoughts & 0.03 & 0.59 \\
Thoughts about dirt, ordering, cleaning & 0.57 & -0.04 \\
Thoughts about mistakes and accidents & 0.47 & 0.31 \\
Aggressive thoughts & -0.06 & 1.03 \\
Thoughts about contamination & 0.73 & -0.02 \\
\hline
\end{tabular}

ROII-20: Revised Obsessive Intrusion Inventory-20

and test-retest reliability indices for the components of the ROII-20 are reported in Table 3. The Cronbach $a$ ranged from 0.76 to 0.91 , suggesting good internal consistency for the subscales. The Cronbach $\alpha$ of the higher-order subscales ( 0.88 for the AOs subscale and 0.80 for the ROs subscale) also suggested good internal consistency. Finally, among participants who completed the ROII- 20 twice, correlations between the two administrations ranged from 0.80 to 0.89 , which suggested good test-retest reliability.

\section{Correlations between the ROII-20 and other measures}

Table 4 reports the correlations between the components of the ROII-20 with the other questionnaires measuring $\mathrm{OCD}$ symptoms, anxiety, and depression. First, the OCI-R total score was positively associated with the scores of all five lower-order subscales and two-higher order subscales (AOs and ROs) of the ROII-20. However, the ROs score was more strongly associated with the OCI-R total score than the AOs score was $(\mathrm{Z}=4.49, \mathrm{p}<0.01)$. Three subscales of the ROs scale were also strongly associated with most of the subscales of the OCI-R. However, the relationships between the two subscales of the AOs scale (i.e., aggressive thoughts and sexual thoughts) and the subscales of the OCI-R were mostly weak or not significant. Second, the PIWUSR-obsessional impulses subscale was strongly associated with the AOs scale and its two subscales, whereas the relationships between the PIWSUR-obsessional impulses scale and the three subscales of the ROs scale were very weak or not significant. Third, both of the AOs and ROs scales were moderately associated with the BAI. However, the correlation between the AOs scale and the PIWSURobsessional impulses subscale was greater than the correlation between the AOs scale and the BAI $(\mathrm{Z}=4.68, \mathrm{p}<0.01)$, and the relationship between the ROs scale and the OCI-R was stronger than the relationship between the ROs scale and the BAI $(\mathrm{Z}=4.47, \mathrm{p}<0.01)$. Finally, the relationships between the AOs/ ROs scales and the CES-D were significant but weak. The AOs scale was more strongly associated with the PIWSUR- 


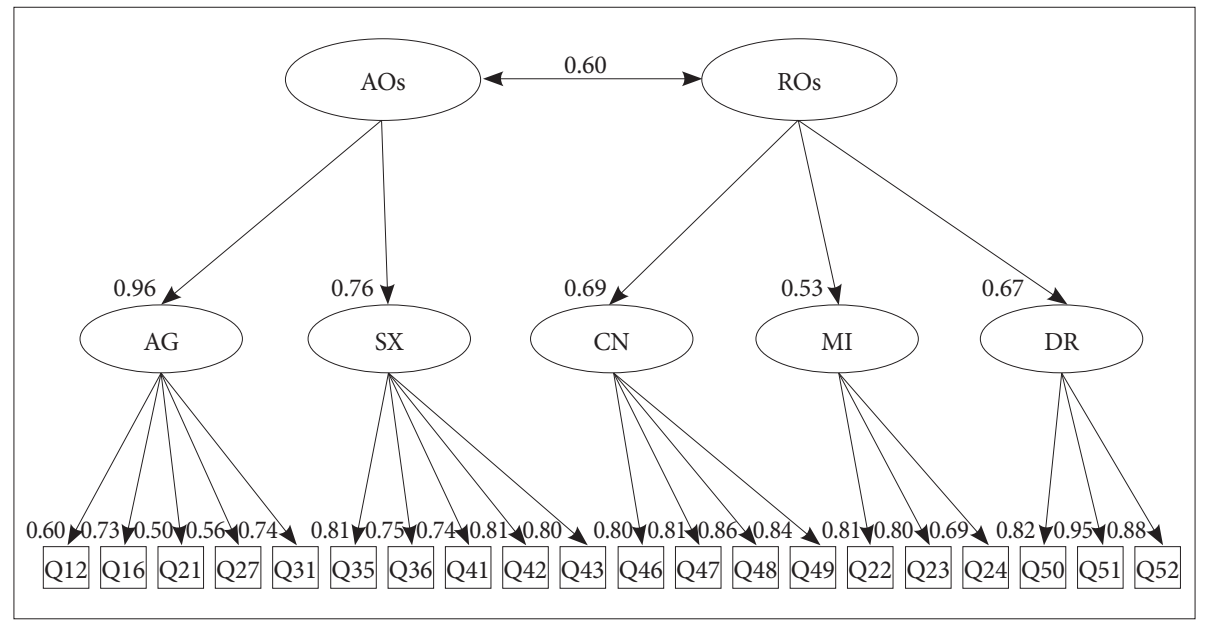

Figure 1. A hierarchical model in which aggressive thoughts and sexual thoughts depend on a common higher order construct of autogenous obsessions; thoughts about contaminations, thoughts about mistakes/accidents, and thoughts about dirt/ordering/cleaning depend on a common higher order construct of reactive obsessions. Ovals reflect latent variables and rectangles reflect manifest variables. Singleheaded arrows reflect factor loadings, whereas double-headed arrows reflect correlations between the latent variables. AOs: autogenous obsessions, ROs: reactive obsessions, AG: aggressive thoughts, SX: sexual thoughts, CN: thoughts about contamination, MI: thoughts about mistakes and accidents, DR: thoughts about dirt/ordering/cleaning.

Table 3. Internal consistency and test-retest reliability of the ROII20 subscales

\begin{tabular}{lcccc}
\hline & Mean & SD & $\alpha$ & Test-retest \\
\hline 1. Aggressive & 1.79 & 2.60 & 0.76 & $0.80^{*}$ \\
2. Sexual & 3.01 & 3.92 & 0.88 & $0.82^{*}$ \\
3. Contamination & 3.76 & 3.97 & 0.87 & $0.86^{*}$ \\
4. Mistakes/accidents & 4.28 & 2.97 & 0.80 & $0.84^{*}$ \\
5. Dirt/ordering/cleaning & 3.81 & 3.82 & 0.91 & $0.89^{*}$ \\
\hline
\end{tabular}

${ }^{*} \mathrm{p}<0.01$. ROII-20: Revised Obsessive Intrusion Inventory-20

obsessional impulses subscale than with the CES-D ( $Z=5.47$, $\mathrm{p}<0.01)$ and the ROs scale's correlation with the OCI-R was significantly higher than its correlation with the CES-D $(\mathrm{Z}=$ $6.28, \mathrm{p}<0.01)$.

\section{DISCUSSION}

The present study examined the psychometric properties of a short version of the Korean adaptation of the ROII developed by Purdon and Clark. ${ }^{2}$ EFAs revealed that a hierarchical model fit the data. The hierarchical model consisted of five lower-order factors (i.e., aggressive thoughts, sexual thoughts, thoughts about contamination, thoughts about mistakes/accidents, and thoughts about dirt/ordering/cleaning) and two higher-order factors (i.e., AOs and ROs). CFA also confirmed that this hierarchical model is appropriate for application to the data obtained. Internal consistency and test-retest reliability of the scale ranged from good to very good. Specific links to psychiatric symptoms were identified. More precisely, the two subtypes of mental intrusions (autogenous and reactive) were related to the OCI-R. However, the associations between
ROs and the OCI-R were stronger than the associations between AOs and the OCI-R. One possible explanation for these differential relationships is that the OCI-R primarily consists of overt OCD symptoms such as washing, ordering, checking, or hoarding. These overt OCD symptoms have been considered to be more closely related to ROs than AOs. ${ }^{6}$ The PIWSUR-obsessional impulses subscale was related to AOs exclusively. This result indicates that the AOs subscale of the ROII-20 exhibits good convergent validity because the PIWSUR-obsessional impulses subscale primarily concerns obsessions about harming oneself or others and obsessions related to sexual behaviors. ${ }^{16}$ Finally, moderate relationships between $\mathrm{AOs} / \mathrm{ROs}$ and anxiety and relatively weak relationships between $\mathrm{AOs} / \mathrm{RO}$ and depression were found, as demonstrated in previous studies. ${ }^{8}$ However, the AOs subscale of the ROII- 20 was more strongly associated with the PIWSURobsessional impulses subscale than with the BAI and the CES-D. The relationship between the ROs subscale and the OCI-R was stronger than the relationship between the ROs subscale and the measures assessing depression and anxiety. Overall, the current study demonstrated that the ROII-20 possesses good psychometric properties.

The completion time of the original 52-item scale is approximately $13 \mathrm{~min}$ (15 seconds per item), whereas the ROII20 takes only $5 \mathrm{~min}$ to complete. This significant time saving is only justified if the psychometric properties of the shortform measure remain largely comparable to those of the original measure. ${ }^{26}$ We found the ROII-20 to have a theoretically based factorial structure. ${ }^{6}$ Moreover, the internal consistency coefficients of the ROII- 20 are similar to those obtained with the original scale devised by Lee and Kwon ${ }^{6}$ (the Cronbach $\alpha$ 
Table 4. Correlations between other measures and the subscales of the ROII-20

\begin{tabular}{clllllll}
\hline & AG & SX & CN & MI & DR & AOs & ROs \\
\hline OCI-R total & $0.28^{* *}$ & $0.24^{* *}$ & $0.48^{* *}$ & $0.49^{* *}$ & $0.48^{* *}$ & $0.30^{* *}$ & $0.60^{* *}$ \\
OCI-R checking & $0.17^{*}$ & $0.20^{* *}$ & $0.34^{* *}$ & $0.54^{* *}$ & $0.36^{* *}$ & $0.21^{* *}$ & $0.49^{* *}$ \\
OCI-R hoarding & $0.17^{* *}$ & $0.22^{* *}$ & $0.31^{* *}$ & $0.39^{* *}$ & $0.26^{* *}$ & $0.23^{* *}$ & $0.39^{* *}$ \\
OCI-R neutralizing & $0.25^{* *}$ & $0.16^{*}$ & $0.28^{* *}$ & $0.32^{* *}$ & $0.28^{* *}$ & $0.23^{* *}$ & $0.36^{* *}$ \\
OCI-R obsessing & $0.27^{* *}$ & $0.21^{* *}$ & $0.18^{* *}$ & $0.28^{* *}$ & $0.24^{* *}$ & $0.27^{* *}$ & $0.28^{* *}$ \\
OCI-R ordering & $0.20^{* *}$ & 0.11 & $0.34^{* *}$ & $0.31^{* *}$ & $0.48^{* *}$ & $0.17^{* *}$ & $0.47^{* *}$ \\
OCI-R washing & $0.17^{* *}$ & $0.15^{*}$ & $0.65^{* *}$ & $0.32^{* *}$ & $0.47^{* *}$ & $0.18^{* *}$ & $0.63^{* *}$ \\
PI-impulses & $0.57^{* *}$ & $0.50^{* *}$ & $0.14^{*}$ & $0.14^{*}$ & 0.12 & $0.60^{* *}$ & $0.17^{*}$ \\
BAI & $0.38^{* *}$ & $0.25^{* *}$ & $0.29^{* *}$ & $0.39^{* *}$ & $0.25^{* *}$ & $0.35^{* *}$ & $0.37^{* *}$ \\
CES-D & $0.19^{* *}$ & $0.25^{* *}$ & $0.17^{*}$ & 0.13 & 0.12 & $0.26^{* *}$ & $0.18^{* *}$ \\
\hline
\end{tabular}

${ }^{*} \mathrm{p}<0.05,{ }^{* *} \mathrm{p}<0.01$. OCI-R: Obsessive Compulsive Inventory-Revised, PI-impulses: Padua Inventory Washington State University Revision: obsessional impulses subscale, BAI: Beck Anxiety Inventory, CES-D: Centers for Epidemiologic Studies Depression Scale, AG: aggressive thoughts, SX: sexual thoughts, CN: thoughts about contamination, MI: thoughts about mistakes and accidents, DR: thoughts about dirt/ordering/cleaning, AOs: Autogenous obsessions, ROs: Reactive obsessions, ROII-20: Revised Obsessive Intrusion Inventory-20

of the AOs and ROs were 0.95 and 0.89 for the original scale and 0.88 and 0.85 for the short-form scale, respectively). Taken together, these results support the use of the ROII-20 for meaningful time saving.

We developed the ROII-20 by retaining the 10 items that loaded most strongly on each of the two factors (AOs and ROs). Although this technique has been frequently used to develop short-form measures, it has the limitation that selected items reflect narrower constructs. ${ }^{26}$ This potential problem is herein discussed with respect to one of the components of the ROII-20: aggressive thoughts. The original ROII contains 31 items tapping various types of aggressive thoughts: aggressive thoughts while driving, thoughts about hurting others, thoughts about hurting family, thoughts about causing a public scene, and thoughts about impulsive damage. ${ }^{2}$ We selected five of these items, which could diminish the breadth of the original measure and omit certain aspects of the construct (i.e., aggressive thoughts). The ROII-20 items with aggressive contents, however, represent all of the major aggressive contents of the original ROII (i.e., two items from 'hurting others', one item from 'hurting family', one item from 'causing a public scene', and one item from 'impulsive damage'), except for only one category (aggressive thoughts while driving).

In the present study, we examined the external validity of the ROII-20 using several measures tapping OCD symptoms and general distress (i.e., depression and anxiety), and we were able to determine the scale's convergent validity and criterion validity. Moreover, we examined the differential relationships between the AOs/ROs subscales and two measures tapping OCD symptoms to determine the subscales' discriminant validity. The discriminant validity of the whole scale, however, has yet to be established. Because mental intrusions are similar to other unwanted cognitions (e.g., worry) ${ }^{27}$ and still different from those cognitions, it would be valuable to examine the discriminant validity of the ROII-20 using wellvalidated measures assessing such cognitions.

\section{REFERENCES}

1. American Psychiatric Association. Diagnostic and Statistical Manual of Mental Disorders: DSM-IV-TR. Washington DC: American Psychiatric Pub; 2000.

2. Purdon C, Clark DA. Obsessive intrusive thoughts in nonclinical subjects. Part I. Content and relation with depressive, anxious and obsessional symptoms. Behav Res Ther 1993;31:713-720.

3. Rachman S, de Silva P. Abnormal and normal obsessions. Behav Res Ther 1978;16:233-248.

4. Salkovskis PM. Cognitive-behavioural factors and the persistence of intrusive thoughts in obsessional problems. Behav Res Ther 1989;27:677682.

5. Rachman S. A cognitive theory of obsessions. Behav Res Ther 1997; 35:793-802.

6. Lee HJ, Kwon SM. Two different types of obsession: autogenous obsessions and reactive obsessions. Behav Res Ther 2003;41:11-29.

7. Seo JW, Kwon SM. Autogenous/reactive obsessions and their relationship with negative self-inferences. J Obsessive Compuls Relat Disord 2013;2:316-321.

8. Lee HJ, Kwon SM, Kwon JS, Telch MJ. Testing the autogenous-reactive model of obsessions. Depress Anxiety 2005;21:118-129.

9. Lee HJ, Telch MJ. Autogenous/reactive obsessions and their relationship with OCD symptoms and schizotypal personality features. J Anxiety Disord 2005;19:793-805.

10. Belloch A, Cabedo E, Carrio C, Larsson C. Cognitive therapy for autogenous and reactive obsessions: clinical and cognitive outcomes at post-treatment and 1-year follow-up. J Anxiety Disord 2010;24:573580 .

11. Besiroglu L, Sozen M, Ozbebit O, Avcu S, Selvi Y, Bora A, et al. The involvement of distinct neural systems in patients with obsessive-compulsive disorder with autogenous and reactive obsessions. Acta Psychiatr Scand 2011;124:141-151.

12. Moulding R, Kyrios M, Doron G, Nedeljkovic M. Autogenous and reactive obsessions: further evidence for a two-factor model of obsessions. J Anxiety Disord 2007;21:677-690.

13. Stanton JM, Sinar EF, Balzer WK, Smith PC. Issues and strategies for reducing the length of self-report scales. Pers Psychol 2002;55:167-194. 
14. Foa EB, Huppert JD, Leiberg S, Langner R, Kichic R, Hajcak G, et al. The obsessive-compulsive inventory: development and validation of a short version. Psychol Assess 2002;14:485-496.

15. Woo CW, Kwon SM, Lim YJ, Shin MS. The Obsessive-Compulsive Inventory-Revised (OCI-R): psychometric properties of the Korean version and the order, gender, and cultural effects. J Behav Ther Exp Psychiatry 2010;41:220-227.

16. Burns GL, Keortge SG, Formea GM, Sternberger LG. Revision of the Padua Inventory of obsessive compulsive disorder symptoms: distinctions between worry, obsessions, and compulsions. Behav Res Ther 1996;34:163-173.

17. Min BB, Won HT. Reliability and validity of the Korean translations of Maudsley Obsessional-Compulsive Inventory and Padua Inventory. Korean J Clin Psychol 1999;18:163-182.

18. Radloff L. The CES-D scale: A self-report depression scale for research in the general population. Appl Psychol Meas 1977;1:385-401.

19. Cho MJ, Kim KH. Use of the Center for Epidemiologic Studies Depression (CES-D) Scale in Korea. J Nerv Ment Dis 1998;186:304-310.

20. Beck AT, Brown G, Epstein N, Steer RA. An inventory for measuring clinical anxiety : psychometric properties. J Consult Clin Psychol 1988;
56:893-897.

21. Yook SP, Kim ZS. A clinical study on the Korean version of Beck Anxiety Inventory : comparative study of patient and non-patient. Korean J Clin Psychol 1997;16:185-197.

22. Rosseel Y. lavaan: An R Package for Structural Equation Modeling. J Stat Softw 2012;48:1-36.

23. Hu LT, Bentler PM. Cutoff criteria for fit indexes in covariance structure analysis: conventional criteria versus new alternatives. Struct Equ Modeling 1999;6:1-55.

24. Schermelleh-Engel K, Moosbrugger H, Müller H. Evaluating the fit of structural equation models: test of significance and descriptive goodness-of-fit measures. Meth Psychol Res 2003;8:23-74.

25. Bentler PM. Comparative fit indexes in structural models. Psychol Bull 1990;107:238-246.

26. Smith GT, McCarthy DM, Anderson KG. On the sins of short-form development. Psychol Assess 2000;12:102-111.

27. Turner SM, Beidel DC, Stanley MA. Are obsessional thoughts and worry different cognitive phenomena? Clin Psychol Rev 1992;12:257270 